\title{
Quantum Calculation of Four-body interaction of Solid Neon
}

\author{
ZHENG Xingrong ${ }^{1, a}$, LIU Xiaojun ${ }^{1, b}$ \\ ${ }^{1}$ College of Electrical Engineering, Longdong University, Qingyang 745000, China \\ ${ }^{a}$ Corresponding author: zhengxingrong2006@163.com, bemail: 454096807@qq.com
}

\begin{abstract}
Keywords: Solid neon, ab initio Hartree-Fock SCF method, Many-body expansion method, Convergence, Cohesive energy, Equations of state

Abstract. Based on the First-principle, using ab initio Hartree-Fock self consistent field method combined with many-body expansion method, two-, three-, and four-body potential energy of face-centered cubic (fcc) solid neon are calculated with the atomic distance R from 1.6 to $3.0 \AA$. Then we obtain the cohesive energy, the zero-point vibration energy and equation of state (EOS). In addition, we discuss the truncation and convergence of many-body potential of solid neon. The numerical results shows that, For a certain atomic distance $\mathrm{R}$, all many-body potential energy tend to a saturation value when the neighboring atoms increases. The even many-body contributions to the cohesive energy, such as two-, four-body terms and so on, are positive, whereas the odd many-body contributions to the cohesive energy, such as two-, four-body terms and so on, are negative. The zero-point vibration energy of solid neon is very small which only is $6 \%$ of the total atomic interaction energy, but should not be neglected. Compared with the exprimental data emphasize the importance of the four-body interactions in compressed solid neon. Only taking into account the two-body term, the pressure is much overestimated, and our calculated results are in good agreement with the experimental values at the low-pressure regions $(<15 \mathrm{GPa})$. Adding three-body term up to $55 \mathrm{Gpa}$. Considering to the four-body term, it have a good consistency at the experimentally studied ranges of pressures $0 \sim 237 \mathrm{GPa}$, and may be helpful to accurately explain the phenomenon of the experiment above $237 \mathrm{Gpa}$ when the higher-body many-body effects are considered.
\end{abstract}

\section{Introduction}

Neon ( $\mathrm{Ne}$ ) has a relatively simple full-shell structure, and then its crystal and electronic structures remain simple at very high pressures, making it is an ideal candidate to test quantum many-body theory under high pressures [1-3]. The study suggests the stability of the fcc phase over a wide pressure range, $\mathrm{Ne}$ is used as a quasi-hydrostatic pressure-transmitting medium [4]. Powder X-ray diffraction experiments of $\mathrm{Ne}$ have been done up to $237 \mathrm{Gpa}$ at room temperature, and it remains the fcc phase at the extremely high pressure [5,6]. Therefore, many significant attention, such as theoretical and experimental studies, have been paid to the study of the many-body potential energy and equation of state for solid $\mathrm{Ne}$ [8-13], and these studies mainly contained research work of two-body potential and three-body potential. But these study shows that the two-body and three-body potentials can only accurately describe the compression properties of solid Ne at low pressure, and they can not accurately describe the compression properties at high pressures, so, the higher-body interactions need to considered.

$\mathrm{Ab}$ initio Hartree-Fock SCF method has become a useful tool for calculating the potential energies of atoms, molecules and the systems consisting of many atoms [14,15]. Here, using this method combined with many-body expansion method, we accurately calculate two-, three- and four-body potentials of fcc solid $\mathrm{Ne}$ at the atomic distance $\mathrm{R}$ from $1.6 \AA$ to $3.0 \AA$, and then we obtain the cohesive energy, the zero-point vibration energy and equation of state of solid Ne. Finally, the truncation and convergence of many-body potential for solid neon are also discussed.

\section{Theoretical model and calculation method}

In a given $(\mathrm{Ne})_{n}$ clusters, the potential energy $V_{n}(o)$ between the central atom $o$ and its neighbours in the crystal lattice can be expressed as [16] 


$$
V_{n}(o, 1,2,3, \ldots, n-1)=E\left(r_{o}, r_{1}, r_{2}, \ldots, r_{n-1}\right)-n E_{0},
$$

where $E_{0}$ is the ground state energy of the isolated atom, $E\left(r_{o}, r_{1}, r_{2}, \ldots, r_{n-1}\right)$ represents the total ground state energy of $(\mathrm{Ne})_{n}$ clusters, and it not only can be obtained by solving the Schrödinger equation [16], but also is calculated by ab initio Hartree-Fock SCF method based on GAMESS Programme [17].

Using many-body expansion method, $V_{n}(o)$ can be also developed into a many-body expansion:

$$
\begin{aligned}
V_{n}(o) & =\sum_{i=1}^{n-1} u_{2}(o, i)+\sum_{i<j=1}^{n-1} u_{3}(o, i, j)+\sum_{i<j<k=1}^{n-1} u_{4}(o, i, j, k)+\cdots \\
& =U_{2}(o)+U_{3}(o)+U_{4}(o)+\cdots
\end{aligned}
$$

where $i, j$ and $k$ denote different neighbours of the central atom $o,(n-1)$ is the total number of considered neighbours. $U_{2}(o), U_{3}(o)$ and $U_{4}(o)$ represent the total two-, three- and four-body interaction energy between atom $o$ and its neighbours, respectively. The two-, three- and four-body potential can be expressed as

$$
\begin{aligned}
& u_{2}(o, i)=E\left(r_{o}, r_{i}\right)-2 E_{0}, \\
& u_{3}(o, i, j)=E\left(r_{o}, r_{i}, r_{j}\right)-3 E_{0}-\sum_{o<i=1}^{3} u_{2}(o, i), \\
& u_{4}(o, i, j, k)=E\left(r_{o}, r_{i}, r_{j}, r_{k}\right)-4 E_{0}-\left(\sum_{o<i<j=1}^{4} u_{3}(o, i, j)+\sum_{o<i=1}^{4} u_{2}(o, i)\right) .
\end{aligned}
$$

Therefore, the cohesive energy $E(V)$ of each atom is a function of volume $V$, which can be expanded to a series of many-body contributions.

$$
\begin{aligned}
E(V) & =E_{2}(V)+E_{3}(V)+E_{4}(V)+\cdots \\
& =\frac{1}{2} U_{2}(o)+\frac{1}{3} U_{3}(o)+\frac{1}{4} U_{4}(o)+\cdots
\end{aligned}
$$

The pressure is a function of volume $V$ and temperature $T$, and it are consist of the many-body pressure, zero-point vibrational pressures and thermal pressures [15].

$$
P(V, T)=P_{2}(V)+P_{3}(V)+P_{z p}(V)+P_{t h}(V, T),
$$

where $P_{z p}$ represents the zero-point vibrational pressure, $P_{t h}$ represents the thermal pressure, and the many-body pressure $P_{n}$ can be expressed by the cohesive energy curves.

$$
P_{n}(V)=-\frac{\partial E_{n}(V)}{\partial V},(n=2,3) .
$$

In the actual calculation, the zero-point vibrational pressure and the thermal pressure are small, therefore, in this paper, we omit the these two parts, and only consider the many-body pressure term.

\section{The results and discussion}

\section{The select of neighboring atoms}

Using many-body expansion method, we calculate the total atomic potential energy and many-body potential energy of fcc solid $\mathrm{Ne}$. The results show that the faster many-body expansion converge, the more the time for the calculation is saved. In addition, we calculate the change of the total atomic potential energy with the increase of the neighboring atoms, as shown in Fig.1. The figure show that, As long as the number of the neighboring atoms are enough, the total potential energy of solid $\mathrm{Ne}$ tend to a convergence value. The main contribution to the total potential energy come from the potential energy between the central atom and 12 atoms of the first neighboring shell. When the atomic distance $R$ decreases, it needs to consider the effects of neighboring atoms of the second, third shells and higher shells. Taking into account the interaction between the central atom and 12 atoms of the first neighboring shell, we obtain accurate atomic potential at $R=3.0 \AA, 2.6 \AA, 2.0 \AA$. While $R=1.6 \AA$, the atomic numbers need to add 6 atoms of the second neighboring shell, the potential energy $V_{n}(o)$ 
generally tend to convergence value. In this work, the computational efficiency is considered, so our calculation only includes 18 atoms of two neighboring shells, which can get a better approximation.

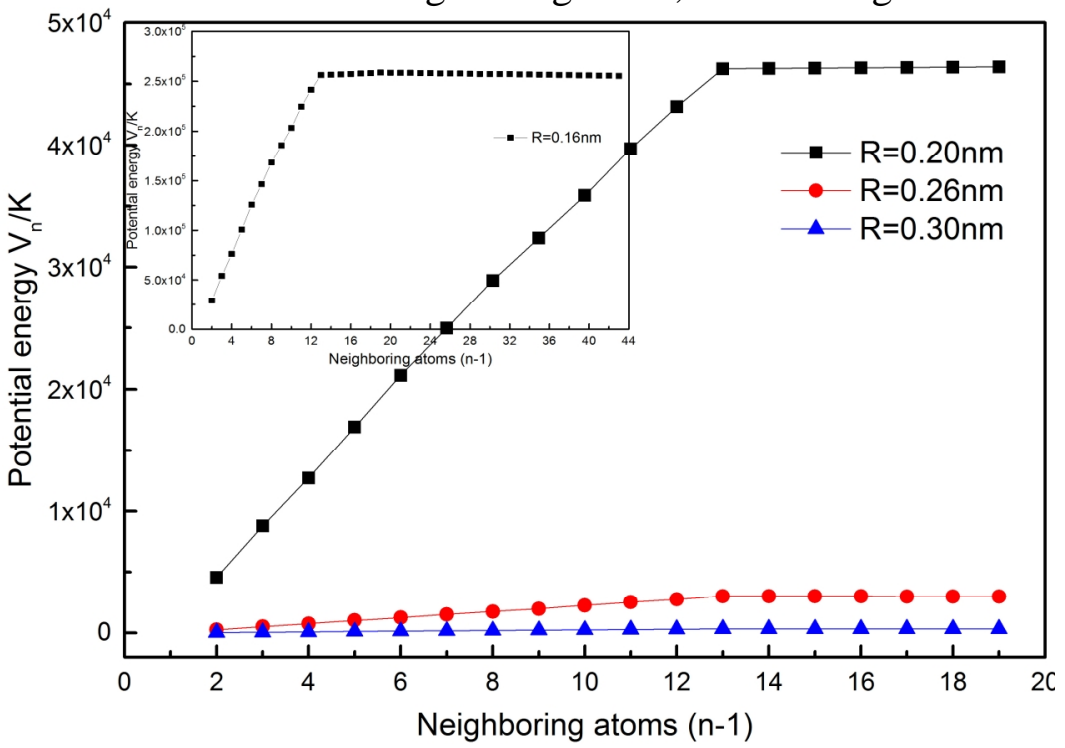

Fig.1 The relation between potential energy and the number of neighboring atoms.

\section{The truncation and convergence of many-body potential}

When the larger number of neighboring atoms is considered, the potential energy of crystal lattice atoms will tend to convergence. For a certain atomic distance $R$, with the increase of the neighboring atoms, this is to say, the effects of the neighboring atoms of the second, third and higher shells are considered, the atomic potential energy $V_{n}(o)$ and many-body potential energy $U_{n}(o)$ of fcc solid $N e$ tend to a convergence value, as shown in Fig.2. We can see from the figure that, taking into account the interaction between the central atom and 12 atoms of the first neighboring shell, it can get a accurate atomic potential at $R=2.6,3.0 \AA$, and $V_{n}(o)$ and $U_{n}(o)$ tend to a convergence value. While $R=2.0 \AA$, the number of atoms need to add 6 atoms of the second neighboring shell, the potential energy $V_{n}(o)$ generally tend to a saturation value. When $R=1.6 \AA, 43$ atoms which include 24 atoms of the third neighboring shell are considered, the four-body potential energy $U_{4}(o)$ and the potential energy $V_{n}(o)$ generally tend to saturation.
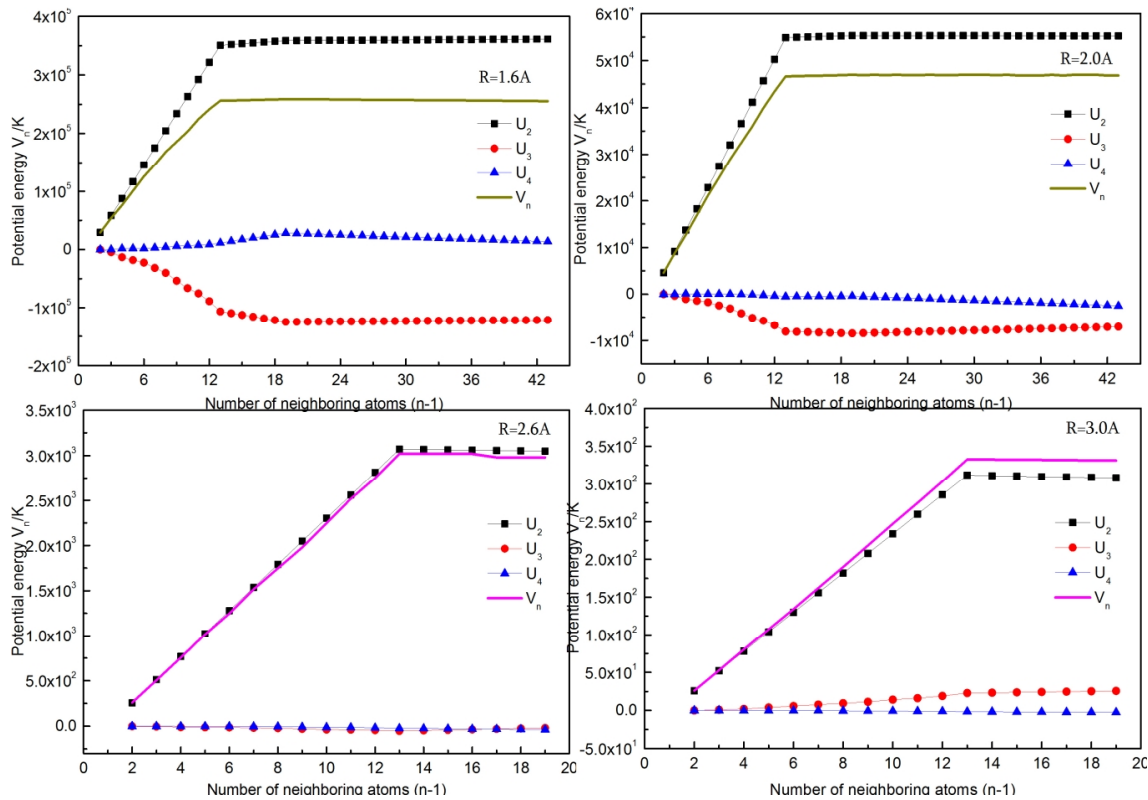

Fig. 2 The variations of the atomic potential erengy $V_{n}(o)$ and its many-body potential energy

$U_{n}(o)$ with the number of neighbouring atoms at $R=1.6,2.0,2.6,3.0 \AA$ in fcc phase. 


\section{The cohesive energy}

Using $a b$ initio Hartree-Fock self consistent field method and Eq. (8), we calculate that two-, threeand four-body contributions to the cohesive energy of fcc solid $N e$ at the atomic distance from $R=1.6 \AA$ to $R=3.0 \AA$, as shown in Fig.3. The numerical results show that the even many-body contributions to the cohesive energy, such as two-, four-body terms and so on, are positive, whereas the odd many-body contributions to the cohesive energy, such as two-, four-body terms and so on, are negative. Only considering the two-body term, the curve of the two-body contribution to the cohesive energy $E_{2}$ and the curve of the total cohesive energy $E_{234}$ are not almost coincident at the larger range of $R$. When the three-body contribution to the cohesive energy $E_{3}$ is considered, the curve of the twoand three-body contribution to the cohesive energy $E_{23}$ and the curve of the total cohesive energy $E_{234}$ are almost coincident at the atomic distance $R>1.8 \AA$. This is to say, the three-body contribution to the cohesive energy $E_{3}$ is very slight and it can be ignored at the atomic distance $R>1.8 \AA$, but $E_{3}$ play an important role when $R<1.8 \AA$, and it can not be neglected in this range. Over a distance range of 1.60 to $1.80 \AA$, the four-body contribution have to be included. In this work, the zero-point vibration energy $E_{z p}$ [19] of solid neon is very small which only is $6 \%$ of the many-body interaction energy, but should not be neglected.

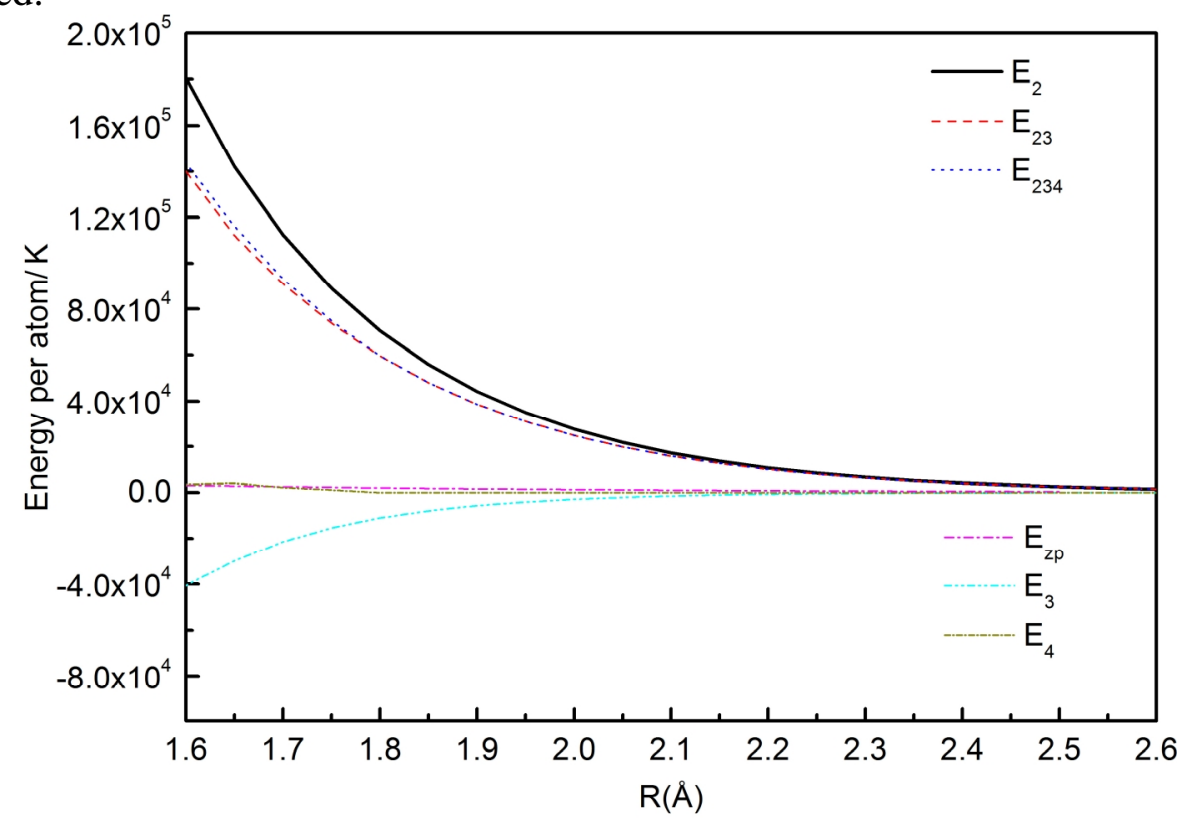

Fig.3 The two-, three- and four-body contributions to the cohesive energy in fcc solid $\mathrm{Ne}$.

\section{Equation of state}

The equation of state of fcc solid $\mathrm{Ne}$ are calculated at room temperature using Eqs.(9), (10), and we make comparisons among the calculated results, the former results [2] and the experimental results $[5,6,18]$, as shown in Fig.4. Compared results show that only taking into account the two-body term, our calculated results and the previous results $[2,5,6,18]$ are in good agreement with the experimental values at $P<10 \mathrm{GPa}$. Considering the two- and three-body terms, the calculated results and the experimental results have a good consistency at high pressure range 10-55GPa. Considering to the four-body term, it have a good consistency at the experimentally studied ranges of pressures 0 237Gpa. When $P>237 \mathrm{GPa}$, it need consider the effect of five- and higher-body terms, and may be helpful to accurately explain the phenomenon of the experiment above $237 \mathrm{Gpa}$ when the effect of five-body, six-body potential pressure are considered. 


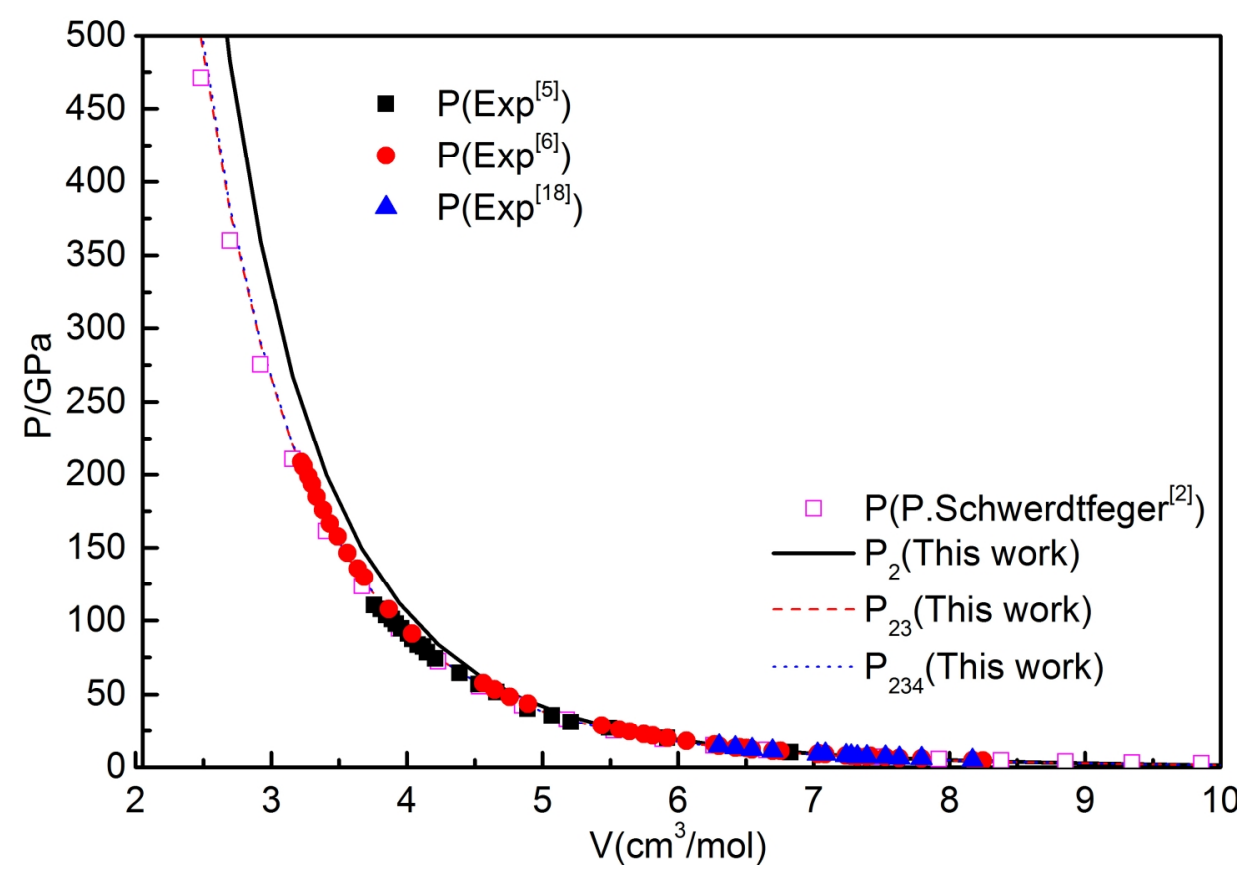

Fig.4 Comparison between the theoretical and experimental equations of state for solid $\mathrm{Ne}$.

\section{Acknowledgement}

In this paper, this work is supported in part by the innovation talents programme fund for the Organization Department of Gansu provincial Party committee (D96).

\section{References}

[1] Pechenik E, Kelson I, Makov G: Phys Rev B. 78(2008), 134109

[2] Schwerdtfeger P, Hermann A: Phys Rev B. 80(2009), 064106

[3] Drummond N D, Needs R J: Phys Rev B. 73(2006), 024107

[4] Tsuchiya T and Kawamura K: J. Chem. Phys. 117(2002), 1030

[5] Hemley R J, et al: Phys. Rev. B. 39(1989), 11820

[6] Dewaele A, et al: Phys Rev B. 77(2008), 094106

[7] K. Takemura, T. Watanuki, K. Ohwada, et al: Journal of Physics. 215(2010), 01201

[8] Wu N, et al: Chinese Journal of High Physics. 26(2012), 41

[9] Aziz R A: High Temp High Press. 12(1980), 565

[10] Aziz R A and Slaman M J: Chem. Phys. 130(1989), 187

[11] Stawomir M. Cybulski, Rafal R. Toczylowski: J.Chem. Phys. 111(1999), 10520

[12] Freiman Y A, Tretyak S M: Low Temp Phys. 33( 2007), 545

[13] Loubeyre P: Phys Rev B. 37(1987), 5432

[14] Cai J, Hu X and Chen N: J. Phys. Chem. 66(2005), 1256

[15] Tian C L, et al: J. Phys.: Condens. Matter. 18(2006), 8103

[16] Tian C L, et al: Chin .Phys. Lett. 20(2003), 706

[17] Information on http://www.msg.ameslab.gov/gamess/

[18] Finger L W, et al: Appl Phys Lett. 39(1981), 892

[19] Zheng X R: Journal of Ningxia university. 37(2016), 429 